\title{
Investigation of protein aggregation dynamics with a Bloch surface wave sensor
}

\author{
Vincent Paeder*, Sara Santi, Valeria Musi, Hans Peter Herzig \\ Ecole Polytechnique Fédérale de Lausanne (EPFL), Institute of Microengineering (IMT), Optics and \\ Photonics Technology Laboratory (OPT), Neuchâtel, Switzerland
}

\begin{abstract}
We present a study of the dynamics of protein aggregation using a common path heterodyne Bloch surface wave sensing scheme. We demonstrate the ability to detect, during thermal incubation, the early events linked to the aggregation of proteins related to conformational diseases. Alzheimer's amyloid- $\beta$ 1-42 is used to demonstrate the efficiency of the method. A model based on elementary interactions is shown to describe accurately the aggregation process. The described sensing scheme is sensitive to the early events of the aggregation process. is hence proposed as a method for the detection of early stages of the evolution of conformational diseases.
\end{abstract}

Keywords: biological sensor, surface waves, protein aggregation

\section{INTRODUCTION}

A recent challenge for biophotonics techniques, already commonly used in sensing applications, is to develop and implement new diagnostic tools in different diseases and clinical settings. Here, we propose a new label-free optical platform based on Bloch Surface Waves ${ }^{1}$ (BSW) sensitive to optical variations caused by conformational changes in proteins. In particular, this innovative and robust technology is applied to a topic that has become increasingly relevant in biomedical research: the early diagnosis of aberrant degenerative pathologies that are caused by the formation of amyloid fibrils (protein aggregates). An increasing number of pathologies (degenerative diseases) result from an incorrect protein folding (misfolding) associated with the formation of aggregates (amyloid fibrils ${ }^{2,3}$ ). Disorders such as Alzheimer and Parkinson diseases, Huntington's chorea, Creutzfeldt-Jacob disease, cystic fibrosis, Gaucher's disease, type II diabetes mellitus and the wide group of systemic amyloidosis are associated with aggregation of misfolded polypeptide chains which are toxic to the cell ${ }^{4}$. Amyloid fibrils, particularly in their prefibrillar forms (precursors), can be highly toxic to cells, suggesting that pre-amyloid oligomeric structures, play a key role in disease pathogenesis ${ }^{5,6}$.

It remains essential to develop new tools allowing early detection of the aggregates, since small oligomeric forms of aggregates are emerging as being the toxic species, suggesting their key role in disease pathogenesis ${ }^{2}$. It is imperative that new forms of diagnostics be developed to detect the disease prior to the onset of clinical symptoms. This will offer patients the prospect of preventive treatments that can block or significantly curb disease progression.

Amyloid fibrils are defined as fibrillar polypeptide aggregates with $\beta$-sheet secondary structure ${ }^{7}$. For many years the only structural information about amyloid fibrils came from imaging techniques (TEM, AFM) and from X-ray fiber diffraction. A range of experiments, indicates that the fibrils have extensive $\beta$-sheet character, and that these sheets run perpendicular to the fibril axis to generate what is described as a cross- $\beta$ structure ${ }^{8}$ (Fig. 1). Understanding why and how proteins may adopt a non-native fold is essential for designing therapeutic approaches ${ }^{9}$.

Alzheimer's disease (AD) is the most common neurodegenerative disorder and a major health concern to societies worldwide. Recent research progress has given detailed knowledge on the molecular pathogenesis of Alzheimer's disease. $\mathrm{AD}$ is associated with senile plaques of extracellular amyloid- $\beta$ protein $(\mathrm{A} \beta)^{11,12}$ and neurofibrillar tangles of filamentous aggregates of abnormally phosphorylated and abnormally $\beta$-folded tau protein ${ }^{13,14}$. AD is caused by an enzymatic cleavage of the amyloid precursor protein (APP), a normal constituent of neuronal cells thought to be important for the neuronal development and function. APP releases a mixture of A $\beta$ peptides, the longest ones being $A \beta_{(1-40)}$ and $A \beta_{(1-42)}$, which are all highly fibrillogenic ${ }^{12}$. A crucial issue in understanding the molecular bases of this disease is the identification of the toxic species.

* vincent.paeder@epfl.ch 
a)

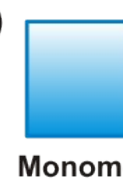

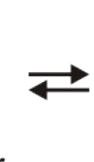
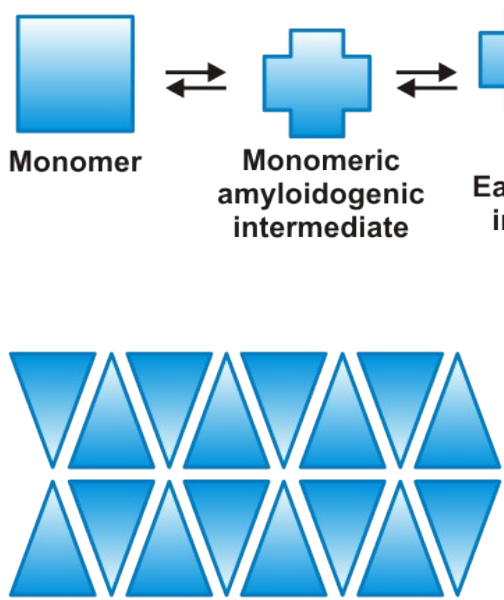

Amyloid fibril

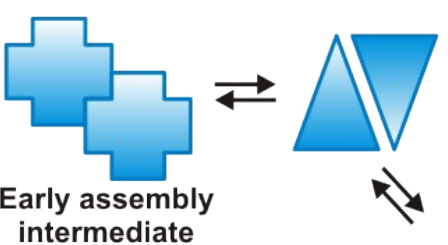

termediate
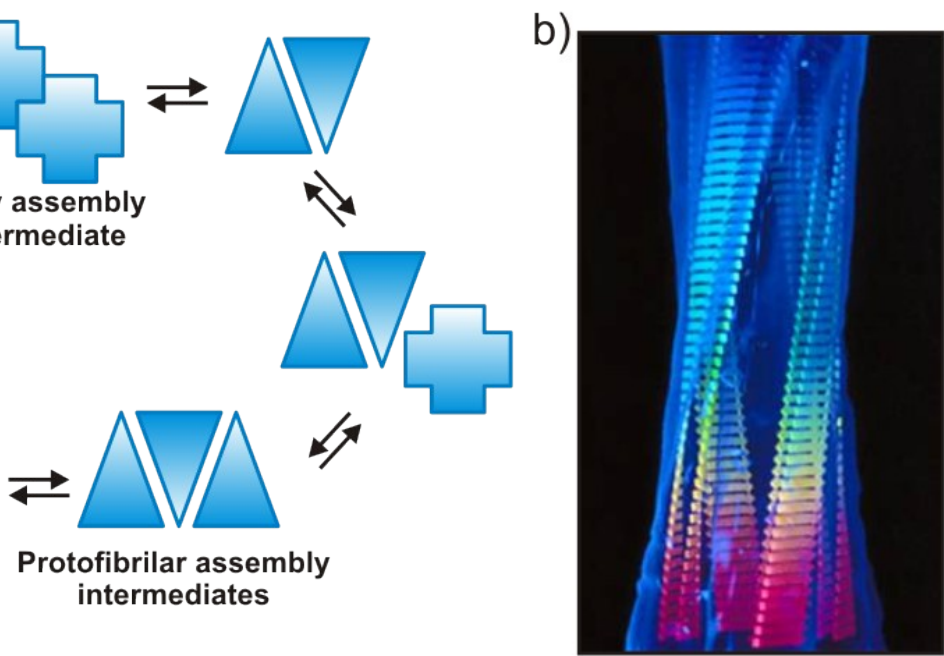

Figure 1. a) Schematic description of the process of amyloid formation ${ }^{2}$ of a full length initially folded protein. The mechanism by which the different proteins gain a conformation, allowing monomers to bind to each other to form the regular amyloid fibril, is under intensive investigation. b) A molecular model of an amyloid fibril. This model is derived from cryo-EM analysis of fibrils and illustrates the polypeptide chain involved in b-sheet conformation ${ }^{10}$.

Currently, no definitive diagnostic test for $\mathrm{AD}$ exists. The presence of neurofibrillary tangles and amyloid deposits are still the disease-defining parameters. Increasing evidence suggests that toxicity is associated with the so-called "soluble toxic oligomers" ${ }^{15-17}$ rather than to the final mature fibers. Much interest has therefore been paid for the study of the oligomers both in $\mathrm{A} \beta$ peptides and tau proteins, directly linked to pathologies ${ }^{18}$ in order to identify reliable biomarkers for $\mathrm{AD}$ that are suitable for minimal invasive early diagnosis and prognosis of $\mathrm{AD}$. Exploring the molecular and pathological basis of $\mathrm{AD}$ can illuminate aspects of the underlying disease mechanism and provides unique opportunities to gain insights into the cascade of pathological events and how they relate to clinical manifestations. In order to significantly tackle the most catastrophic and devastating symptom of Alzheimer's dementia, we must be able to detect the disease prior to the onset of clinical symptoms, and be able to offer patients preventative treatments that block or significantly slow disease progression ${ }^{19}$. In vitro studies on these aggregates should provide a description of the fibril formation mechanism at the very early stages of aggregation ${ }^{20}$.

Here, we briefly describe the recent results obtained on the conformational analysis and aggregation kinetics study of $\mathrm{A} \beta_{(1-42)}$ peptides, the first protein system used for our experiments. The peptide aggregation during thermal incubation $\left(\mathrm{pH} 7,37^{\circ} \mathrm{C}\right)$ has been monitored using several well-established techniques available in the laboratory (size exclusion chromatography, UV-visible, fluorescence and FTIR spectroscopies). The calibration of the biosensor is made using lysozyme protein, responsible for the hereditary non-neuropathic systemic amyloidosis. This protein has known structure and folding properties, it has been well characterized and shown to be useful as model system to examine structural and mechanistic principles. Conclusions drawn on these molecules could therefore be confidently generalized to all amyloid fibrils.

\section{EXPERIMENTAL METHOD}

The applied detection scheme is based on a common path heterodyne interferometer including a BSW sensor ${ }^{21}$ (Fig. 2). TE and TM polarizations are separated after the sensor and used as reference and object channels, respectively. Such a configuration is allowed by the polarization-sensitive characteristics of the BSW, providing a high stability and a high sensitivity. The sensor is mounted on a rotating stage to track the resonance frequency of the BSW over large dynamic ranges. 
BSW are surface waves supported at the surface of truncated periodic structures. A surface mode with a propagation constant below the light line in the external medium can be supported provided that a forbidden band exists in the underlying structure. Forbidden bands are well-known features of periodic structures. Therefore, a BSW can be supported by

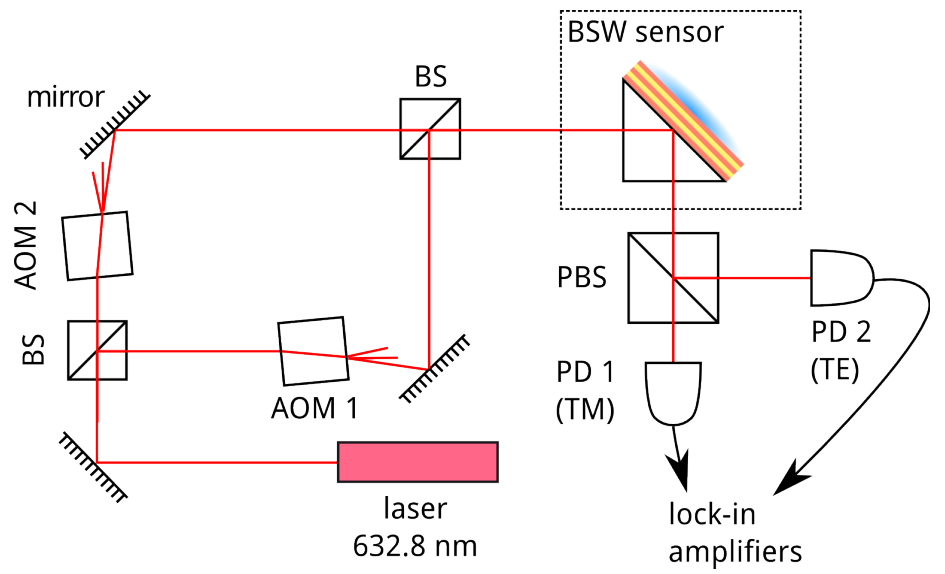

Figure 2. Principle of the common path heterodyne BSW sensing scheme

For the sake of convenience and simplicity, the structure used here is a $1 \mathrm{D}$ dielectric multilayer stack made of $\mathrm{SiO}_{2}$ (refractive index $\mathrm{n}_{\mathrm{L}}=1.46$ ) and $\mathrm{Si}_{3} \mathrm{~N}_{4}$ (refractive index $\mathrm{n}_{\mathrm{H}}=1.95$ ), and grown by PECVD (Oxford Plasmalab 80+). The designed stack supports a surface mode in TM polarization in an aqueous environment $(\mathrm{n}=1.333)$.

The BSW is excited in a Kretschmann configuration (Fig. 3). The configuration used in our experiment corresponds to case a) (vertical configuration). This choice of configuration allows to discriminate the aggregation process from the interaction of proteins with the sensor surface. It is however less sensitive to the precipitation of aggregates than configuration $\mathrm{b}$ ). The best configuration depends on the complexity of the aggregation process and of the affinity of the sensor surface to proteins in one or several states of aggregation.

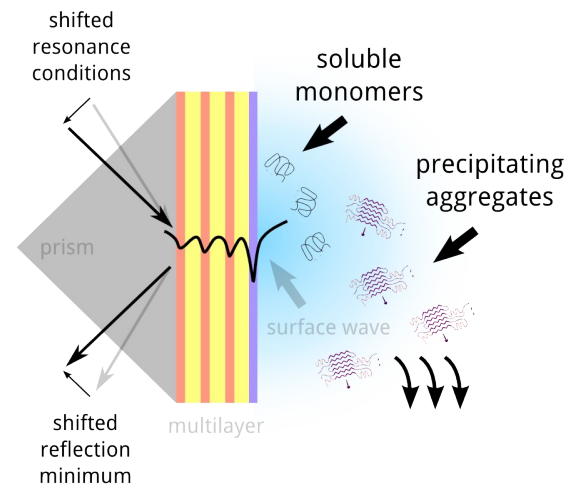

a)

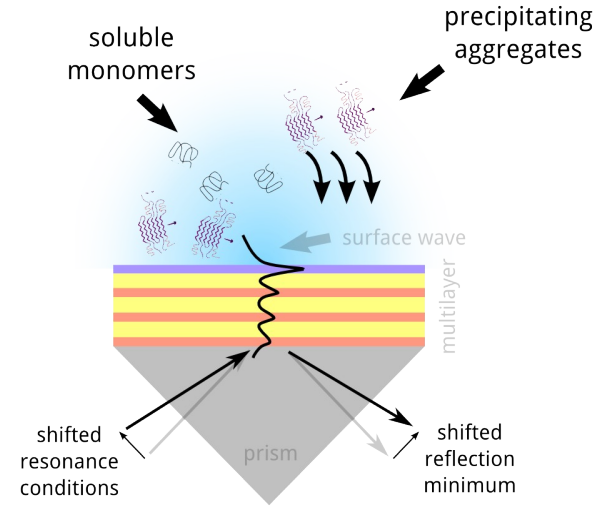

b)

Figure 3. Principle of excitation of a BSW in Kretschmann configuration showing two modes of operation: a) vertical configuration; the aggregates precipitate, causing a concentration depletion near the sensor surface, and b) horizontal configuration; the aggregates precipitate on the surface, causing a local concentration increase.

A temperature controlled microfluidic chamber is mounted atop the multilayer to incubate small amounts of solution. The microfluidic chamber is made of an UV curable polymer (Norland Adhesives NOA-61) replicated with a PDMS stamp from a microstructure fabricated in SU-8. Mechanically roughened teflon connectors are attached to the input and output channels with a flexible UV curable polymer (Norland Adhesive NOA-68). During incubation, to avoid the evaporation of the fluid, the input and output connectors are sealed with paraffin. 
The sensor is mounted in a $\theta-2 \theta$ configuration on rotating stages. The resonance angle corresponds to a sharp dip in the reflection spectrum. The evolution of the aggregation process is recorded over time by monitoring the position of the reflectivity minimum. Additionally, the phase of the reflected beam is obtained thanks to the heterodyne setup. Near resonance, the phase variations are much larger than the intensity variations. The phase signal enables hence to improve the sensitivity of the sensing scheme.

\section{PREPARATION AND CHARACTERISATION OF PROTEIN SAMPLES}

The purification of $A \beta(1-42)$ samples has been carried out by size exclusion chromatography (SEC), following the protocol as developed by A. Jan and H. Lashuel ${ }^{23,24}$. The A $\beta$ monomeric samples were purified and separated from the oligomeric species by choosing specific buffer conditions (Fig. 2a). The conformational characterization of monomeric or aggregated forms and the determination of the content of $\beta$-sheet secondary structure during the incubation have been carried out using FTIR spectroscopy measurements (Fig. 2b). During the last years the use of Fourier transform infrared (FTIR) spectroscopy has dramatically expanded and become crucial for monitoring the structure of proteins and to detect the presence of $\beta$-sheet secondary structure. Typically, the sample is examined before and after aggregation and an increase in $\beta$-sheet secondary structure is observed upon amyloid fibril formation ${ }^{25-27}$.

Moreover, the kinetics of aggregation have been monitored by UV-visible spectroscopy (Fig. 2c) and fluorescence spectroscopy by the Thioflavin T (ThT) colorimetric assay (Fig. 2d). The monomeric samples were incubated under specific conditions ( $\mathrm{pH}$, temperature, and ionic strength) responsible for oligomer and fibril formation. The results were then compared with the BSW based biosensor analysis of the samples incubated under the same conditions.
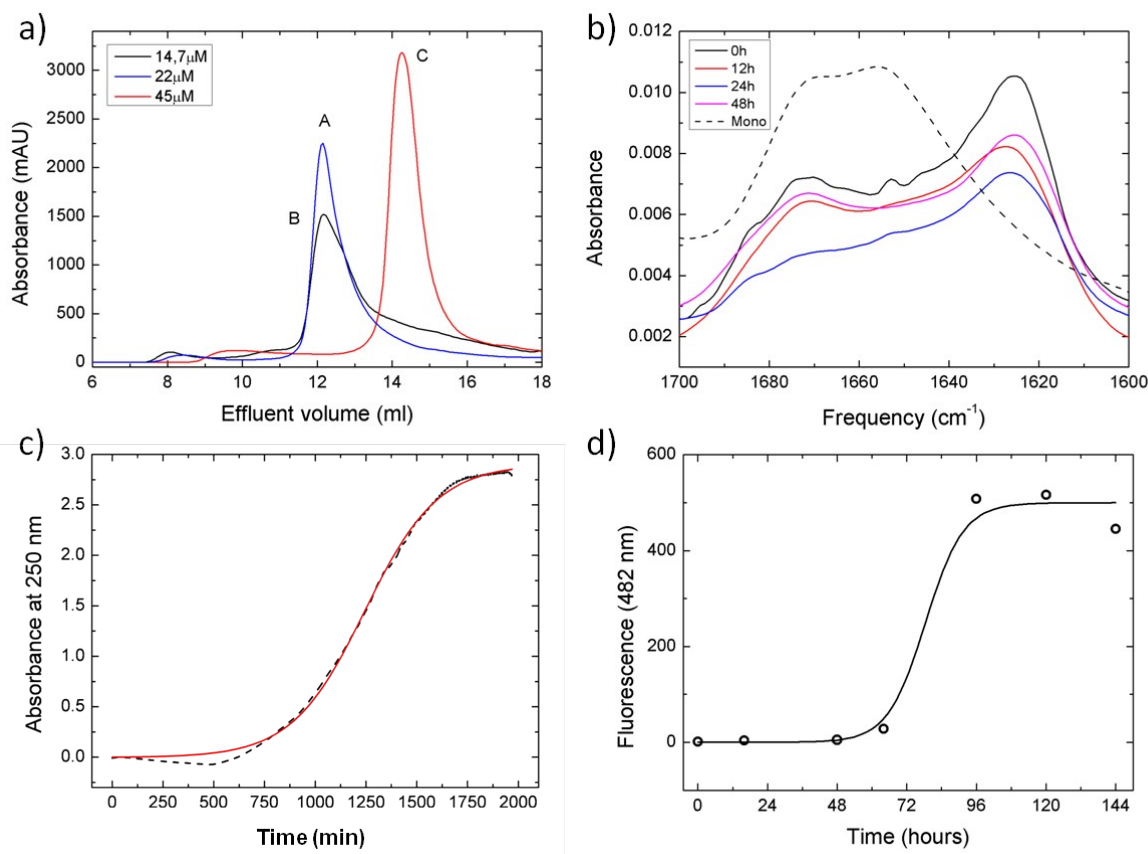

Figure 4. Biochemical and biophysical characterization of the $A \beta_{(1-42)}$ peptide. (a) Size exclusion chromatography. The samples $\mathrm{A} \beta_{(1-42)}$ are purified to monomer conformation by size exclusion chromatography in different buffer composition. In blue and black line runs of $\mathrm{A} \beta$ with elution buffer $10 \mathrm{mM}$ Tris $\mathrm{HCl}$ at different concentration $\left(\mathrm{Vr} \mathrm{A} \beta_{(1-42)}\right.$ oligomer 8.15 $\mathrm{mL}$; $\operatorname{Vr~A} \beta_{(1-42)}$ monomer $12.53 \mathrm{~mL}$ ); in red line running of $\mathrm{A} \beta$ with elution buffer $10 \mathrm{mM}$ Tris $\mathrm{HCl}$ and $70 \mathrm{mM} \mathrm{NaCl}$ $\left(\operatorname{Vr~A} \beta_{(1-42)}\right.$ oligomer $9.4 \mathrm{~mL}$; $\operatorname{Vr~A} \beta_{(1-42)}$ monomer $14.5 \mathrm{~mL}$ ). (b) FTIR spectroscopy. Amide I band region in the FTIR spectrum of $A \beta$ peptide. Solid lines: IR spectra of the $A \beta_{(1-42)}$ peptide during the incubation in D2O. Dotted line: the IR spectrum of a monomer obtained in different buffer conditions $(30 \% \mathrm{v} / \mathrm{v}$ HFIP, $70 \% \mathrm{v} / \mathrm{v}$ D2O). (c) UV-vis spectroscopy. Time-dependent absorbance of incubated $\mathrm{A} \beta_{(1-42)}$ sample monitored at $250 \mathrm{~nm}$ (black line). Red line: fitting curve obtained applying the nucleation-elongation kinetic model ${ }^{28}$. (d) Fluorescence. Time-dependent aggregation of $A \beta_{(1-42)}$ monitored by the fluorescence change of Thioflavin- $T$ at $482 \mathrm{~nm}$. Open circles represent fluorescence data, the continuous black line is the curve obtained by fitting the experimental data with the equation describing the nucleation-elongation kinetic model $^{28}$. 
The dominant contribution to the BSW sensor signal comes from density changes near the sensor surface. Such density changes happen in various situations, such as:

- $\quad$ proteins are adsorbed on the surface, causing a concentration increase

- aggregates grow in immediate proximity to the surface, causing a concentration increase

- $\quad$ aggregates precipitate away from the surface, causing a concentration decrease

Based on these considerations, an aggregation model based on elementary interactions has been derived (Fig. 5). The protein-protein interactions are taken form the model proposed in ref. 28 . The interactions with the sensor surface depend on the hydrophobicity of the surface ${ }^{22}$.

Three different concentrations of $A \beta_{(1-42)}$ have been investigated. The sensor response in the three cases is shown in Fig. 6. The model corresponding to the assumptions proposed above is seen to be in very good agreement. According to these assumptions, a strong signal is observed in the early stages of the aggregation process. The BSW sensing scheme is hence proposed as a tool to detect the aggregation of proteins at early stages.

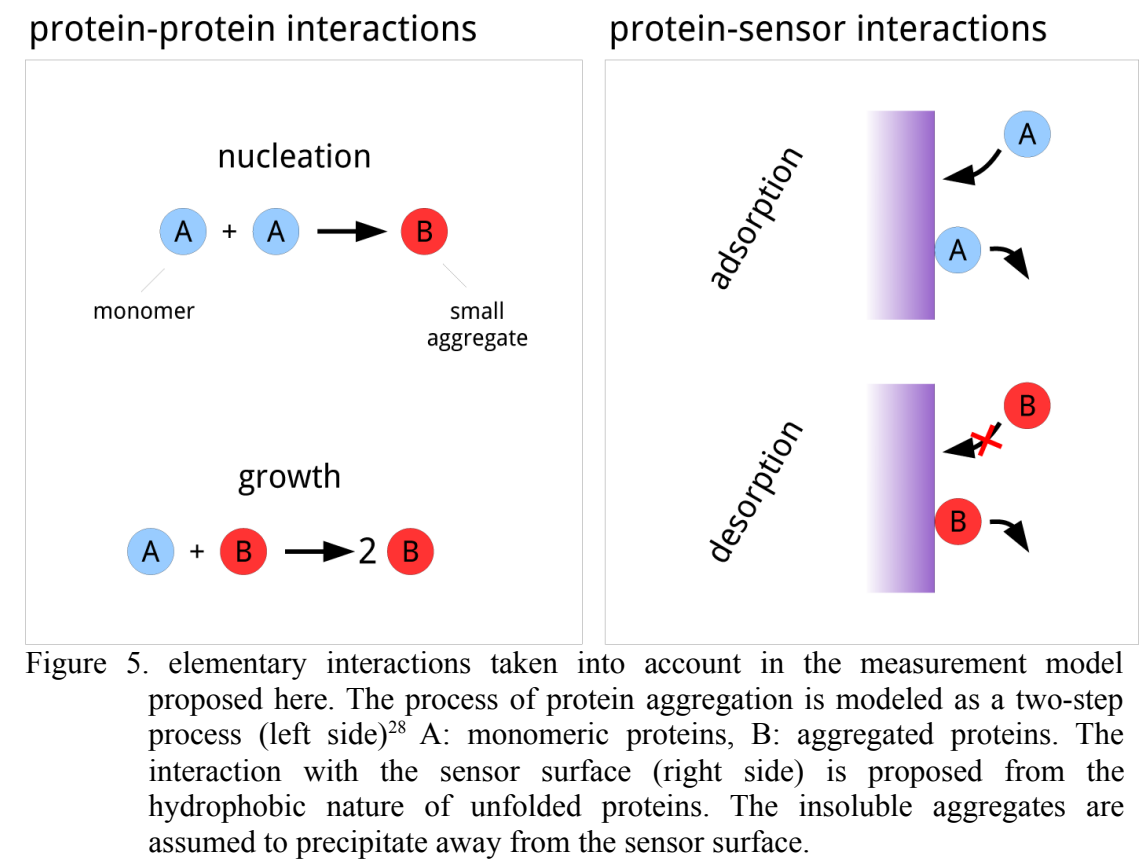

\section{CONCLUSIONS}

We show in this study that a BSW-based heterodyne sensing scheme is suitable to investigate the dynamics of the aggregation processes of proteins. Measurements on Alzheimer's beta amyloids show that the detection process relies on adsorption of monomers and small aggregates, as well as density depletion due to precipitation of large aggregates. A model based on elementary interactions is proposed. This model is in excellent agreement with the measured data. The ability of the BSW sensing principle is demonstrated to detect the conformational changes of aggregating proteins in a label-free way. These results are of prime interest for the detection of conformational diseases at an early stage. 

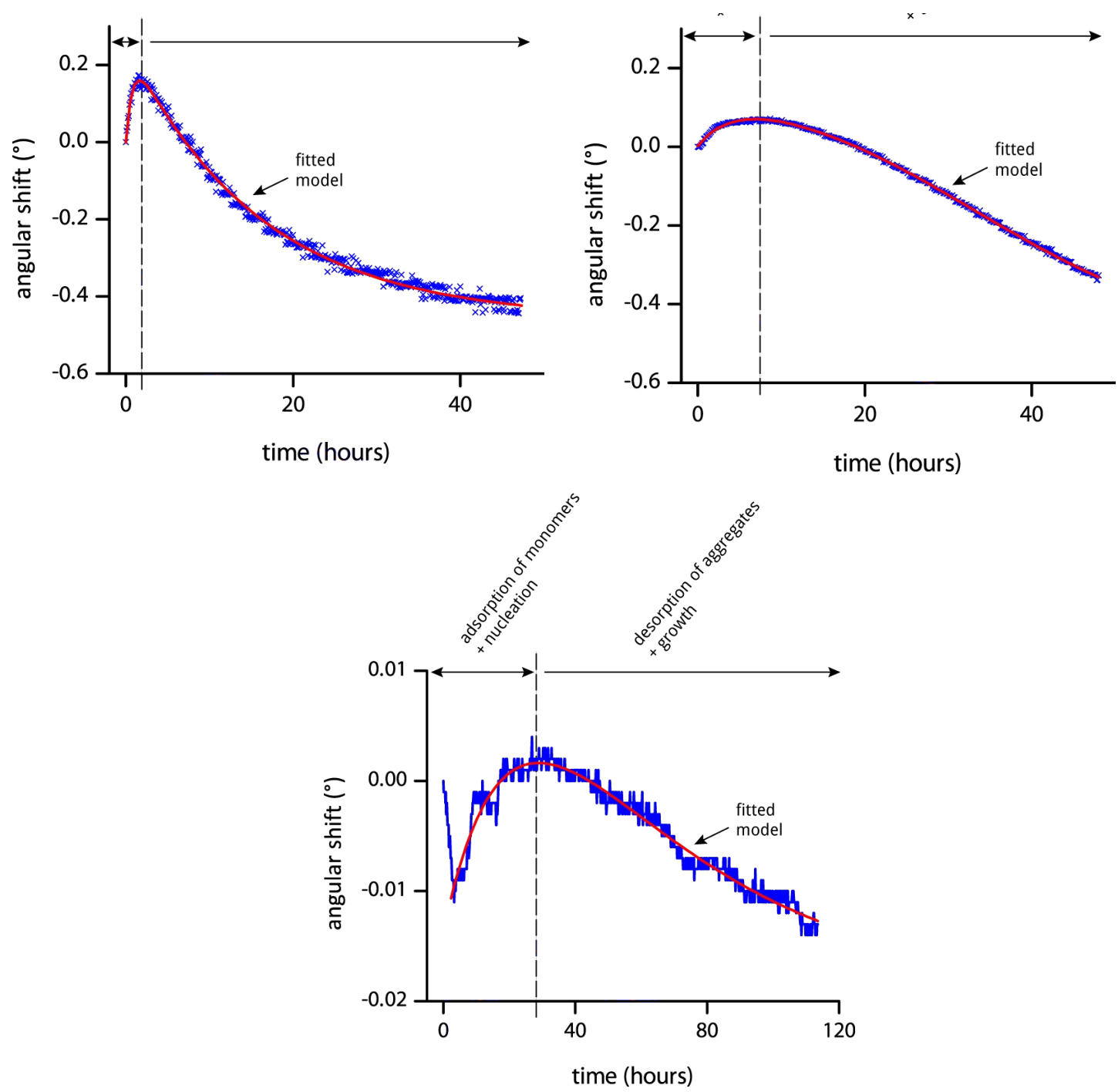

Figure 6. Recorded evolution of the reflectivity minimum for three protein concentrations. a) $45 \mu \mathrm{M}$, b) $22 \mu \mathrm{M}$ and c) $14 \mu \mathrm{M}$. The blue curve corresponds to the measured data and the red curve represents the fitted model.

\section{REFERENCES}

[1] F. Giorgis et al, "Experimental determination of the sensitivity of Bloch Surface Wave based sensors," Opt. Express 18, 8087-8093 (2010)

[2] J.W. Kelly, "Towards an understanding of amyloidogenesis," Nat Struct Biol 9, 323-325 (2002) 
[3] F. Chiti and C.M. Dobson, "Protein misfolding, functional amyloid, and human disease," Ann. Rev. Biochem. 75, 333-366 (2006)

[4] C.G. Glabe, "Structural classification of toxic amyloid oligomers," J Biol Chem 283, 29639-29643 (2008)

[5] P.T. Lansbury and H.A. Lashuel, "A century-old debate on protein aggregation and neurodegeneration enters the clinic," Nature 443, 774-779 (2006)

[6] R. Kayed et al, "Common structure of soluble amyloid oligomers implies common mechanism of pathogenesis," Science 300, 486-489 (2003)

[7] M. Fändrich, "On the structural definition of amyloid fibrils and other polypeptide aggregates," Cell Mol Life Sci 64, 2066-2078 (2007)

[8] O.S. Makin, L.C. and Serpell, "Structures for amyloid fibrils," FEBS J 272, 5950-5961 (2005)

[9] T. K. Chaudhuri, and S. Paul, "Protein-misfolding diseases and chaperone-based therapeutic approaches," FEBS J 273, 1331-1349 (2006)

[10] C. M. Dobson, "Principles of protein folding, misfolding and aggregation," Semin Cell Dev Biol 15, 3-16 (2004)

[11] C. Haass et al, "Normal cellular processing of the beta-amyloid precursor protein results in the secretion of the amyloid beta peptide and related molecules," Ann N Y Acad Sci 695, 109-116 (1993)

[12] M. D. Kirkitadze, and A. Kowalska, "Molecular mechanisms initiating amyloid beta-fibril formation in Alzheimer's disease," Acta Biochim Pol 52, 417-423 (2005)

[13] J. Berriman et al, "Tau filaments from human brain and from in vitro assembly of recombinant protein show cross-beta structure," Proc Natl Acad Sci USA 100, 9034-9038 (2003)

[14] L. I. Binder et al, “Tau, tangles, and Alzheimer's disease,” Biochim Biophys Acta 1739, 216-223 (2005)

[15] M. P. Lambert et al, "Diffusible, nonfibrillar ligands derived from Abeta1-42 are potent central nervous system neurotoxins," Proc Natl Acad Sci USA 95, 6448-6453 (1998)

[16]D. M. Walsh et al, "Naturally secreted oligomers of amyloid beta protein potently inhibit hippocampal longterm potentiation in vivo," Nature 416, 535-539 (2002)

[17] C. G. Glabe, "Common mechanisms of amyloid oligomer pathogenesis in degenerative disease," Neurobiol Aging. 27, 570-575 (2006)

[18] L. C. Serpell, "Alzheimer's amyloid fibrils: structure and assembly," Biochim Biophys Acta 1502, 16-30 (2000)

[19] D. W. Shineman, and H. M. Fillit, "Novel strategies for the prevention of dementia from Alzheimer's disease," Dialogues in clinical neuroscience 11, 129-134 (2009)

[20] S. Baglioni et al, "Prefibrillar amyloid aggregates could be generic toxins in higher organisms," J Neurosci 26, 8160-8167 (2006)

[21] L.-C. Su et al, "Detection of prostate-specific antigen with a paired plasma wave biosensor," Anal. Chem. 82, 3714-3718 (2010)

[22] S. Choi, et al, "Surface plasmon resonance protein sensor using Vroman effect," Biosensors and Bioelectronics 24, 893-899 (2008)

[23] A. Jan et al, "A $\beta 42$ neurotoxicity is mediated by ongoing nucleated polymerization process rather than by discrete A $\beta 42$ species", J. Biol Mol 286, 8585-8596 (2011)

[24] A. Jan et al, "Preparation and characterization of toxic A beta aggregates for structural and functional studies in Alzheimer's disease research," Nat Protoc 5, 1186-1209 (2010)

[25] M. Calero, and M. Gasset, "Fourier transform infrared and circular dichroism spectroscopies for amyloid studies," Methods Mol Biol 299, 129-151 (2005)

[26] H. Hiramatsu, and T. Kitagawa, "FT-IR approaches on amyloid fibril structure," BBA-Proteins and Proteomics 1753, 100-107 (2005)

[27] G. Zandomeneghi et al, "FTIR reveals structural differences between native $\beta$-sheet proteins and amyloid fibrils," Protein Science 13, 3314-3321 (2004)

[28] M. A. Watzky et al, "Fitting yeast and mammalian prion aggregation kinetic data with the Finke-Watzky twostep model of nucleation and autocatalytic growth," Biochemistry 47, 10790-10800 (2008) 